\title{
Valoración de la formación del profesorado: comparativa entre autoformación y formación presencial
}

\author{
Assessment of teacher training: comparison between self-training \\ and face-to-face instruction
}

\author{
Carmen Aneas-Novo \\ Centro del Profesorado de Málaga (España) \\ ORCID: https://orcid.org/0000-0003-1204-1136 \\ carmen.aneas@cepmalaga.com
}

\section{NOTA BIOGRÁFICA}

Doctora en Educación y Comunicación Social por la Universidad de Málaga y Licenciada en Ciencias Ambientales por la Universidad de Granada. Profesora de Enseñanza Secundaria y titular de las especialidades de Biología y Geología y Educación Física. Máster Universitario en Políticas y Prácticas de Innovación Educativa por la Universidad de Málaga; y otros tres Másteres en Prevención de Riesgos Laborales en las especialidades de Seguridad en el Trabajo, Higiene Industrial, y Ergonomía y Psicosociología Aplicada. Mi actividad investigadora queda plasmada en la publicación de varios libros y artículos científicos publicados en revistas de impacto. Actualmente desempeño las funciones de la asesoría de formación del ámbito científico-técnico en el Centro del Profesorado de Málaga y soy Presidenta del Comité de Investigación de Situaciones de Acoso en la Delegación Territorial de Educación, Deporte, Igualdad, Políticas Sociales y Conciliación en Málaga.

José Sánchez-Rodríguez

Universidad de Málaga (España) ORCID: https://orcid.org/0000-0003-4525-8761 josesanchez@uma.es

\section{NOTA BIOGRÁFICA}

Maestro de Educación Infantil y Primaria desde el año 1981. Licenciado en Pedagogía en 1988 por la Universidad de Málaga (UMA), efectuó la lectura de su tesis doctoral en 1999, con una calificación de «sobresaliente cum laude». Destinado durante 4 años en la Dirección de Enseñanza Virtual y Laboratorios Tecnológicos de la UMA realizando labores de formación y asesoramiento al profesorado de dicha Universidad. Fue profesor de la UMA en el Área de Educación Infantil y Nuevas Tecnologías desde noviembre de 1996 hasta diciembre de 2000. Desde enero de 2002 hasta agosto de 2004 fue profesor del Departamento de Teoría e Historia de la Educación. Desde noviembre de 2007 hasta junio de 2009 profesor en el Departamento de Métodos de Investigación e Innovación Educativa y, desde junio de 2009 hasta la fecha de hoy, profesor del Departamento de Didáctica y Organización Escolar de la Facultad de Ciencias de la Educación de la UMA.

Enrique Sánchez-Rivas

Universidad de Málaga (España)

ORCID: https://orcid.org/0000-0003-2518-2026 enriquesr@uma.es

\section{NOTA BIOGRÁFICA}

Doctor en Pedagogía. Profesor en el Departamento de Teoría e Historia de la Educación de la Universidad de Málaga. Su labor investigadora ha sido prolífera. Pertenece al Grupo de investigación Innoeduca. Ha 
GAPP. Nueva Época - N. ${ }^{2}$ 21, Mayo-Octubre 2019 - ISSN: 1989-8991 - DOI: 10.24965/gapp.v0i21.10569 - [Págs. 94-108]

Valoración de la formación del profesorado: comparativa entre autoformación y formación presencial

Carmen Aneas-Novo / José Sánchez-Rodríguez / Enrique Sánchez-Rivas

dirigido dos investigaciones con subvención pública, y ha participado en otras muchas. Todas enmarcadas en las líneas de igualdad y tecnología educativa, sobre las que ha profundizado en estancias de investigación internacionales. Fruto del trabajo de investigación y de divulgación del conocimiento, es autor de una veintena de artículos publicados en revistas de impacto en los últimos diez años, a los que hay que sumar ocho libros. Ha participado como ponente en congresos de ámbito nacional e internacional. Su trabajo en el aula ha sido reconocido con el prestigioso Premio Santillana de experiencias educativas.

\title{
RESUMEN
}

En Andalucía, actualmente, las políticas educativas en materia de formación del profesorado apuestan por la autoformación como eje principal de su planificación en detrimento de la formación presencial. Este artículo ofrece una comparativa entre ambas modalidades con la intención de determinar cuál es la opción mejor valorada por los docentes. En el estudio han colaborado 1.596 profesores que durante el curso 2017/2018 participaron en 105 actividades diferentes en el centro del profesorado (CEP) de Málaga, donde completaron un cuestionario de valoración general de la actividad elaborado y validado ad hoc. Los resultados respaldan la dirección de las estrategias adoptadas en formación del profesorado en dicha comunidad, dado que la autoformación constituye la modalidad con mejor valoración; y entre las razones principales están: que los participantes perciben su mayor aplicabilidad, el apoyo de las ponencias externas, la metodología, los contenidos y el fomento del trabajo colaborativo en este tipo de actividades.

\section{PALABRAS CLAVE}

Aprendizaje; Centro de Profesores; Formación Continua; Formación de Profesores.

\begin{abstract}
In Andalusia, at present, the educational policies in the field of teacher training opt for self-training as the main axis of their planning to the detriment of face-to-face training. This article offers a comparison between both modalities with the intention of determining which option is best valued by the teachers. The study involved the collaboration of 1596 teachers who, during the 2017/2018 academic year, participated in 105 different activities in the «centro del profesorado» (CEP) of Malaga, where they completed a general assessment questionnaire for the activity prepared and validated ad hoc. The results support the optimal direction of the strategies adopted in teacher training in said community, given that self-training is the modality with the best evaluation; and among the main reasons that are perceived by the participants: its greater applicability, the support of the external reports, the methodology, the contents and the promotion of the collaborative work in this type of activities.
\end{abstract}

\section{KEYWORDS}

Learning; Lifelong learning; Teacher Centre; Teacher Education.

\section{SUMARIO}

1. INTRODUCCIÓN. 2. METODOLOGÍA. 2.1. MUESTRA. 2.2. INSTRUMENTOS DE RECOGIDA Y ANÁLISIS DE DATOS. 3. ANÁLISIS Y DISCUSIÓN DE RESULTADOS. 3.1. PREGUNTAS EN ESCALA LIKERT DEL CUESTIONARIO. 3.2. PREGUNTAS ABIERTAS DEL CUESTIONARIO. 4. CONCLUSIONES. 5. REFERENCIAS BIBLIOGRÁFICAS.

\section{INTRODUCCIÓN}

Hoy día la formación permanente ocupa uno de los ejes principales de trabajo en toda la Unión Europea; ello se debe al reconocimiento del aprendizaje como un proceso acumulativo por el que las personas adquieren nuevos conocimientos con los que abordan sus situaciones profesionales, personales y sociales (Díaz, 2016; Valle, 2017). Como señala A. Pérez (2017), en el escenario mundial frente a la incertidumbre de los nuevos tiempos, los docentes deben estar en continua formación para poder provocar en sus discentes el desarrollo de las capacidades necesarias para afrontar las demandas del futuro que está por venir. 
La preocupación por la formación y el perfeccionamiento del profesorado no es una inquietud solo de este tiempo, tiene un largo recorrido histórico en España y en particular en Andalucía, remontándose a los movimientos de renovación pedagógica, pasando por la creación de los CEP en los años 80 (Costa, 2015), hasta nuestros días con la aprobación del Decreto 93/2013 donde se regula la formación inicial y permanente del profesorado en esta Comunidad Autónoma. Dicho Decreto determina que las acciones formativas deberán estar basadas en el análisis, la reflexión y la mejora de la práctica docente, convirtiendo al aula y al centro en escenarios de formación mediante estrategias que estimulen el trabajo cooperativo y el intercambio profesional, la difusión del conocimiento para la creación de redes profesionales y el aprendizaje a través de las buenas prácticas; el legislador señala la autoformación como el modelo formativo más eficaz para alcanzar dichos objetivos. De la misma manera, el III Plan Andaluz de Formación Permanente del Profesorado (2014), propone priorizar actuaciones formativas que se materialicen a través de la autoformación en sus dos modalidades: formación en centros (FC) y grupos de trabajo (GT); e igualmente en la Resolución de 12 de septiembre de 2017 también se apuesta por la autoformación. Esta opción formativa es concebida en Andalucía como un proceso en el que el profesorado se forma en una temática concreta y aprende enseñando a través de su propia práctica, generando nuevos conocimientos mediante el trabajo colaborativo, la investigación y la reflexión individual y compartida; dicho proceso está apoyado por las asesorías de los CEP y por expertos ponentes que de manera paralela apoyan y guían el trabajo de los docentes tanto en la FC como en los GT. Anualmente la Dirección General del Profesorado y Gestión de Recursos Humanos de la Consejería de Educación, publica las instrucciones con las directrices de participación y funcionamiento de FC y GT. En dichas instrucciones la FC es contemplada como una iniciativa de formación vinculada al proyecto educativo de un centro y dirigida a dar respuesta a las necesidades formativas del claustro, en otras palabras, está orientada a la búsqueda de soluciones específicas para mejorar la práctica docente y los resultados del alumnado. La participación en esta modalidad supone un compromiso colectivo, de al menos la mitad del claustro, con los objetivos propuestos en el proyecto de actuación, la realización de tareas con incidencia directa en el aula y en el centro y la utilización de estrategias de trabajo colaborativo las cuales se deberán detallar en cada proyecto de FC (Instrucción 17/2017). Los GT determinan una opción formativa igualmente enfocada a la práctica, asociada a contextos específicos que posibilitan dar respuesta a diferentes niveles de experiencia profesional. Se trata pues de un proyecto de trabajo colaborativo, en el que participan de 3 a 10 participantes, generando un espacio para la reflexión, el intercambio, la construcción conjunta de aprendizajes y la innovación (Instrucción 18/2017).

En consonancia con lo anterior, señala Marcelo (1999) que las Administraciones educativas plantean la autoformación como un instrumento para el desarrollo de su política y sus necesidades; asumir que la formación del profesorado ha de surgir de los centros supone claramente una apuesta por la profesionalización docente y por la autonomía de las escuelas. En la sistematización de investigaciones sobre el asunto aparece un estudio realizado por Barquín (2014) del que se desprende que en Andalucía las modalidades de GT y FC se presentan como las más elegidas por los docentes andaluces. Los autores consultados (García et al, 2001; Imbernón, 1996; Martín, 2005; Murillo y Krichesky 2018; R. Pérez, López, Peralta \& Municio, 2000; Santos, 2010) confirman que el éxito de este tipo de acciones formativas radica en trasladar la formación desde los CEP a los centros educativos para contextualizarla y crear relaciones de cohesión y trabajo colaborativo que favorecerán el proceso de aprendizaje docente. A este respecto, apunta Imbernón (2017) que la profesión docente se construye durante toda la vida profesional y en esta construcción es primordial la relación con los otros, la cultura de centro y el intercambio de prácticas. Desde esta perspectiva, el centro educativo debe convertirse en el espacio por excelencia para la formación del profesorado a través del trabajo cooperativo y la cohesión del claustro (Santos, 2010). Bandeira (2001) caracteriza a las escuelas eficaces señalando que el desarrollo profesional docente se debe fomentar en programas de formación dirigidos desde y hacia la propia escuela. De igual modo, señala Imbernón $(1996,2006)$ que la FC es la formación centrada en la institución educativa, lo que implica trasladar la formación a los centros escolares e iniciar en ellos proyectos de cambio en los que el profesorado pasa a ser protagonista de su propia formación. La autoformación implica superar la dependencia del profesorado que asiste a las formaciones presenciales esperando recibir recetas universales que les muestren qué camino seguir y donde los ponentes interaccionan unidireccionalmente con los formados en el mismo espacio y tiempo. En este contexto la mejora de la formación y del desarrollo profesional implica reconocer al profesorado como verdaderos agentes de cambio social, planificadores y gestores de la enseñanza-aprendizaje, lo que conlleva intervenir en los sistemas que conforman sus funciones laborales (Imbernón, 2012). Se debe concebir al docente como un investigador de 
su práctica diaria, de su propia enseñanza entendida como un proceso de investigación reflexivo y crítico, basado en la detección de problemas relevantes para la práctica y la formación, orientado a la construcción de alternativas para abordar estas situaciones (Elliot, 2010). También, Imbernón (2002) sostiene que la investigación-acción constituye un proceso de formación permanente que parte del análisis de problemáticas reales, promueve la participación, estimula el trabajo en grupo, facilita la toma de decisiones, potencia la capacidad autoformativa y estimula la flexibilidad organizativa. En el estudio realizado por Martín (2005) sobre autoformación, se subraya que el profesorado elige esta opción por sus ventajas con respecto a otro tipo de actividades formativas; el profesorado muestra alta satisfacción al confirmar que mejora y potencia sus relaciones personales y profesionales y les proporciona herramientas para enseñar y mejorar su acción educativa. En otra investigación más reciente realizada por Jarauta, Colén, Barredo y Bozu (2014) sobre la formación permanente del profesorado en Cataluña, se resalta la necesidad de potenciar la formación del profesorado mediante modalidades más participativas, como la FC y los GT.

En el contexto andaluz, los resultados de las investigaciones citadas en los párrafos anteriores han contribuido a un cambio en la percepción de la autoformación, dejando esta de ser una cuestión meramente pedagógica o técnica para convertirse en un asunto central de las políticas relativas a formación permanente del profesorado. No obstante, dichos estudios, si bien respaldan la autoformación, no recogen a plenitud análisis cuantitativos que justifiquen que los docentes también se inclinan en el mismo sentido que la Administración. En consecuencia, este trabajo tiene por objeto analizar de forma comparativa, la valoración general que realizan los participantes en actividades de autoformación y formación presencial. Como hipótesis de trabajo partimos de la premisa de que la primera tiene una valoración mejor por parte de los formados.

\section{METODOLOGÍA}

El presente artículo de investigación se realiza desde la perspectiva de una metodología cuantitativa de tipo exploratorio y de índole longitudinal (Hernández, Fernández y Baptista, 2014). Se centra en analizar las respuestas de los participantes a un cuestionario de valoración general de la actividad en las modalidades de autoformación y formación presencial.

\subsection{Muestra}

El procedimiento de selección de la muestra de estudio fue no probabilístico (Hernández et al., 2014). Los criterios empleados fueron: 1) la temporalización, formaciones que tuvieron lugar entre septiembre de 2017 y mayo de 2018; 2) la modalidad de formación, FC, GT y CP; y, 3) la selección de 35 actividades de cada una de las modalidades previstas.

Para llevar a cabo el cálculo del margen de error de la muestra se consideró como población al profesorado que participó en una formación en el CEP de Málaga en el curso 2016/2017, un total de 7.573 personas. Para este volumen de población, y conforme a las diferentes estratificaciones previstas, se seleccionó una muestra de 1.676 personas que participaban en 105 actividades formativas, con un nivel de confianza del $95 \%\left(Z^{2} a=1,96\right)$ y un margen de error del $2,1 \%$. Tras recoger los datos se obtuvieron 1.596 encuestas válidas lo que dejaba el margen de error en el 2,2\%. La tabla 1 muestra su distribución de acuerdo con los criterios de estratificación señalados.

TABLA 1. DISTRIBUCIÓN FINAL DE LA MUESTRA SEGÚN CRITERIOS DE ESTRATIFICACIÓN

\begin{tabular}{|c|c|c|}
\hline \multirow{4}{*}{ MODALIDAD } & GRUPO DE TRABAJO & 276 \\
\hline & FORMACIÓN EN CENTRO & 501 \\
\hline & CURSO PRESENCIAL & 819 \\
\hline & Total & 1.596 \\
\hline
\end{tabular}

Fuente: Elaboración propia.

A continuación, la tabla 2 muestra el perfil del profesorado que realizó el cuestionario: 
GAPP. Nueva Época - N. ${ }^{2}$ 21, Mayo-Octubre 2019 - ISSN: 1989-8991 - DOI: 10.24965/gapp.v0i21.10569 - [Págs. 94-108]

Valoración de la formación del profesorado: comparativa entre autoformación y formación presencial Carmen Aneas-Novo / José Sánchez-Rodríguez / Enrique Sánchez-Rivas

TABLA 2. COMPOSICIÓN DE LA MUESTRA

\begin{tabular}{c|c|cccc}
\hline \multicolumn{2}{c}{ MODALIDAD } & GT & FC & CP & Total \\
\hline \multirow{2}{*}{ SEXO } & Hombre & 51 & 108 & 249 & 408 \\
\cline { 2 - 6 } & Mujer & 225 & 393 & 570 & 1.188 \\
\hline \multirow{3}{*}{ EDAD } & Media & 42,28 & 44,44 & 44,85 & 44,27 \\
\cline { 2 - 6 } & Desv. típ. & 7,679 & 9,119 & 8,224 & 8,473 \\
\hline
\end{tabular}

Fuente: Elaboración propia.

Debemos detenernos un momento para analizar la composición de la muestra por sexos dado que en este parámetro encontramos la primera gran diferencia entre autoformación y CP. Las diferencias en los recursos humanos andaluces son de un $33,2 \%$ de hombres frente a un $66,8 \%$ de mujeres; en la provincia de Málaga es un $32,9 \%$ frente a un $67,1 \%$, respectivamente (Estadísticas - Recursos Humanos del Sistema Educativo en Andalucía - Consejería de Educación, 2018). Podemos observar que en CP los porcentajes de participación de hombres y mujeres son similares a las distribuciones poblacionales señaladas anteriormente, concretamente $30,4 \%$ de hombres frente al $69,6 \%$ de mujeres; sin embargo, en autoformación dicha participación se inclina del lado de las mujeres con un $80 \%$ frente al $20 \%$ de hombres.

\subsection{Instrumentos de recogida y análisis de datos}

Para la recogida de información se empleó el cuestionario Valoración general de la actividad formativa, elaborado y validado ad hoc (Aneas, Sánchez y Sánchez, 2018). Este instrumento incluye un apartado inicial para recoger datos dirigidos a determinar el perfil profesional del participante; una batería de 18 ítems, en escala Likert de cero a diez, agrupados en 6 dimensiones; y, finalmente, dos preguntas abiertas orientadas a conocer cuáles son los aspectos mejor valorados y los que podrían mejorar en cada caso, con las que matizar y comprender los resultados medidos en las escalas.

Este cuestionario tiene como finalidad medir la satisfacción global de cada participante con la actividad formativa en el momento en el que la finaliza, atendiendo a la adecuación de la organización, los contenidos, el desarrollo de esta, la actuación del ponente y de la asesoría, encargada de organizar la formación, y la satisfacción general y los aprendizajes logrados con la formación. Tras un primer análisis factorial exploratorio se obtuvieron seis dimensiones que explicaban el $78 \%$ de la varianza del modelo con una fiabilidad del 0.882 (Alfa de Cronbach). La dimensión «A. Organización de la actividad formativa» se compone de tres ítems, encaminados a determinar la idoneidad del calendario, el horario, el lugar y los recursos formativos. La dimensión «B. Contenidos de la formación» está integrada por dos ítems, dirigidos a conocer el grado de interés y dificultad de los contenidos. La dimensión «C. Desarrollo de la formación» se conforma por tres ítems, enfocados a establecer el grado en que se ha fomentado el trabajo colaborativo, el nivel de interacción entre sus miembros y la idoneidad de la metodología empleada. En la dimensión «D. Actuación del o la ponente» a través de tres ítems se confiere magnitud a la capacidad para dominar, comunicar y transmitir contenidos del ponente y a su capacidad para fomentar el aprendizaje activo. La dimensión «E. Papel de la asesoría» se compone de dos ítems, para medir cómo perciben los participantes el adecuado diseño de la actividad realizado por la asesoría, y la agilidad de esta para resolver incidencias durante la formación. La última dimensión «F. Satisfacción general y aprendizajes logrados» incluye un total de cinco ítems, donde se considera la adecuación de la actividad a sus expectativas, el grado de utilidad para la práctica profesional, el nivel de aprendizaje adquirido, la satisfacción con la formación y una pregunta dirigida a determinar en qué medida recomendarían a otros participar en una actividad similar.

En CP, el cuestionario fue facilitado a los asistentes el último día de la formación a través un código QR para acceder al mismo. En autoformación, dado que esta formación se prolonga durante todo el curso académico y finaliza el 31 de mayo, los participantes contaron con una semana previa a esta fecha para cumplimentar los cuestionarios.

Los datos obtenidos para los ítems medidos en escala Likert se exportaron al programa estadístico SPSS 22.0 para su análisis. De forma preliminar se realizó un estudio exploratorio de los datos recopilados con la intención de excluir todos los valores perdidos o fuera de rango. El estudio estadístico llevado a cabo 
GAPP. Nueva Época - N. ${ }^{2}$ 21, Mayo-Octubre 2019 - ISSN: 1989-8991 - DOI: 10.24965/gapp.v0i21.10569 - [Págs. 94-108]

Valoración de la formación del profesorado: comparativa entre autoformación y formación presencial

Carmen Aneas-Novo / José Sánchez-Rodríguez / Enrique Sánchez-Rivas

fue de tipo descriptivo e inferencial. Los análisis se realizaron de forma general confrontando la formación presencial con la autoformación.

\section{ANÁLISIS Y DISCUSIÓN DE RESULTADOS}

\subsection{Preguntas en escala Likert del cuestionario}

Los resultados obtenidos con la aplicación del cuestionario han ofrecido la percepción global acerca de la satisfacción del profesorado que finaliza una actividad de alguna de las modalidades formativas seleccionadas para el comparativo objeto de este estudio.

En la tabla 3 se muestran las puntuaciones medias de los ítems que se integran en cada dimensión, diferenciando en la modalidad de autoformación los resultados para GT y FC.

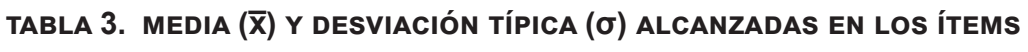

\begin{tabular}{|c|c|c|c|c|c|c|c|c|}
\hline \multirow{3}{*}{$\begin{array}{l}\text { MODALIDAD FORMATIVA } \\
\text { ÍTEM }\end{array}$} & \multicolumn{4}{|c|}{ AUTOFORMACIÓN } & \multirow{2}{*}{\multicolumn{2}{|c|}{$\mathrm{CP}$}} & \multirow{2}{*}{\multicolumn{2}{|c|}{ TOTAL }} \\
\hline & \multicolumn{2}{|c|}{ GT } & \multicolumn{2}{|c|}{ FC } & & & & \\
\hline & $\overline{\mathbf{x}}$ & $\sigma$ & $\overline{\mathbf{x}}$ & $\sigma$ & $\overline{\mathbf{x}}$ & $\sigma$ & $\overline{\mathbf{x}}$ & $\sigma$ \\
\hline $\begin{array}{l}\text { 1. El calendario y el horario de reuniones } \\
\text { y ponencias: }\end{array}$ & 8,12 & 1,98 & 7,92 & 2,03 & 8,05 & 1,81 & 8,02 & 1,91 \\
\hline $\begin{array}{l}\text { 2. El lugar de celebración de reuniones } \\
\text { y ponencias: }\end{array}$ & 8,63 & 1,55 & 8,56 & 1,54 & 7,95 & 1,98 & 8,26 & 1,81 \\
\hline $\begin{array}{l}\text { 3. Los recursos formativos disponibles } \\
\text { (bibliografía, TIC, etc.): }\end{array}$ & 8,16 & 1,68 & 7,98 & 2,05 & 7,63 & 2,20 & 7,83 & 2,08 \\
\hline $\begin{array}{l}\text { 4. Indica el grado de interés de los contenidos } \\
\text { para la docencia: }\end{array}$ & 8,92 & 1,72 & 8,50 & 1,64 & 8,23 & 1,86 & 8,44 & 1,79 \\
\hline $\begin{array}{l}\text { 5. Señala el grado de dificultad } \\
\text { de los contenidos: }\end{array}$ & 6,36 & 2,64 & 5,72 & 3,00 & 5,91 & 2,84 & 5,93 & 2,86 \\
\hline $\begin{array}{l}\text { 6. En qué medida la formación fomenta } \\
\text { el trabajo colaborativo: }\end{array}$ & 8,37 & 2,01 & 8,01 & 2,04 & 7,52 & 2,14 & 7,82 & 2,11 \\
\hline $\begin{array}{l}\text { 7. Nivel de interacción alcanzado entre } \\
\text { los participantes: }\end{array}$ & 8,64 & 1,44 & 8,17 & 1,87 & 7,40 & 2,23 & 7,86 & 2,06 \\
\hline $\begin{array}{l}\text { 8. Señala el grado de idoneidad } \\
\text { de la metodología utilizada: }\end{array}$ & 8,59 & 1,74 & 8,27 & 1,67 & 7,77 & 2,09 & 8,07 & 1,94 \\
\hline $\begin{array}{l}\text { 9. Grado en que el o la ponente domina } \\
\text { los contenidos: }\end{array}$ & 8,89 & 1,95 & 8,97 & 1,43 & 8,81 & 1,74 & 8,87 & 1,69 \\
\hline $\begin{array}{l}\text { 10. Capacidad para comunicar y transmitir } \\
\text { del o de la ponente: }\end{array}$ & 8,87 & 1,92 & 8,78 & 1,72 & 8,59 & 1,85 & 8,70 & 1,83 \\
\hline 11. Fomento del aprendizaje activo: & 8,68 & 1,98 & 8,60 & 1,72 & 8,22 & 2,08 & 8,42 & 1,97 \\
\hline $\begin{array}{l}\text { 12. ¿En qué medida se ha realizado un diseño } \\
\text { adecuado de la actividad? }\end{array}$ & 8,13 & 2,23 & 8,15 & 1,81 & 7,82 & 2,01 & 7,98 & 2,00 \\
\hline $\begin{array}{l}\text { 13. Indica el grado de agilidad en la resolución } \\
\text { de incidencias durante la formación: }\end{array}$ & 8,28 & 2,26 & 8,22 & 1,95 & 7,97 & 2,12 & 8,10 & 2,09 \\
\hline $\begin{array}{l}\text { 14. ¿En qué medida la formación ha respondido } \\
\text { positivamente a tus expectativas iniciales? }\end{array}$ & 8,43 & 1,94 & 7,93 & 2,17 & 7,87 & 2,08 & 7,99 & 2,09 \\
\hline $\begin{array}{l}\text { 15. Grado de utilidad de la formación } \\
\text { para tu práctica profesional: }\end{array}$ & 8,62 & 1,91 & 7,98 & 2,14 & 8,00 & 2,14 & 8,10 & 2,12 \\
\hline $\begin{array}{l}\text { 16. Autoevalúa el nivel de aprendizaje } \\
\text { que has logrado con esta formación: }\end{array}$ & 8,25 & 1,76 & 7,69 & 1,95 & 7,77 & 2,02 & 7,83 & 1,96 \\
\hline
\end{tabular}


GAPP. Nueva Época - N. ${ }^{2}$ 21, Mayo-Octubre 2019 - ISSN: 1989-8991 - DOI: 10.24965/gapp.v0i21.10569 - [Págs. 94-108]

Valoración de la formación del profesorado: comparativa entre autoformación y formación presencial Carmen Aneas-Novo / José Sánchez-Rodríguez / Enrique Sánchez-Rivas

\begin{tabular}{|c|c|c|c|c|c|c|c|c|}
\hline \multirow{3}{*}{$\begin{array}{c}\text { MODALIDAD FORMATIVA } \\
\text { ÍTEM }\end{array}$} & \multicolumn{4}{|c|}{ AUTOFORMACIÓN } & \multirow{2}{*}{\multicolumn{2}{|c|}{$\mathrm{CP}$}} & \multirow{2}{*}{\multicolumn{2}{|c|}{ TOTAL }} \\
\hline & \multicolumn{2}{|c|}{ GT } & \multicolumn{2}{|c|}{ FC } & & & & \\
\hline & $\bar{x}$ & $\sigma$ & $\bar{x}$ & $\sigma$ & $\overline{\mathbf{x}}$ & $\sigma$ & $\overline{\mathbf{x}}$ & $\sigma$ \\
\hline $\begin{array}{l}\text { 17. Grado de satisfacción con la acción } \\
\text { formativa realizada }\end{array}$ & 8,53 & 1,84 & 8,04 & 2,09 & 7,97 & 2,09 & 8,09 & 2,06 \\
\hline $\begin{array}{l}\text { 18. ¿En qué medida recomendarías } \\
\text { a tus compañeros/as participar } \\
\text { en una actividad como ésta? }\end{array}$ & 8,66 & 2,02 & 8,20 & 2,19 & 8,04 & 2,27 & 8,20 & 2,21 \\
\hline
\end{tabular}

Fuente: Elaboración propia.

Se aprecian puntuaciones ligeramente mayores en autoformación que en $\mathrm{CP}$, y más concretamente en los GT. En la tabla 4 se ofrecen los resultados de los valores medios de las dimensiones del cuestionario.

TABLA 4. MEDIA ( $\bar{X})$ Y MEDIANA (MDN) DE LAS DIMENSIONES DE CADA MOdALIDAD FORMATIVA

\begin{tabular}{lcccccc}
\hline \multicolumn{1}{c}{ MODALIDAD } & \multicolumn{2}{c}{ AUTOFORMACIÓN } & \multicolumn{2}{c}{$\begin{array}{c}\text { CURSO } \\
\text { PRESENCIAL }\end{array}$} & \multicolumn{2}{c}{ Global } \\
\hline \multicolumn{1}{c}{ DIMENSIÓN } & $\overline{\mathbf{x}}$ & $\mathbf{M d n}$ & $\overline{\mathbf{x}}$ & $\mathbf{M d n}$ & $\overline{\mathbf{x}}$ & Mdn \\
\hline \multicolumn{1}{c}{ A) Organización de la actividad formativa } & 8,21 & 8,30 & 7,88 & 8,00 & 8,04 & 8,30 \\
\hline B) Contenidos de la formación & 7,35 & 8,00 & 7,08 & 7,00 & 7,21 & 8,00 \\
\hline C) Desarrollo de la formación & 8,29 & 8,70 & 7,56 & 8,00 & 7,92 & 8,30 \\
\hline D) Actuación del ponente o la ponente & 8,79 & 9,00 & 8,54 & 9,00 & 8,66 & 9,00 \\
\hline E) Papel de la asesoría & 8,19 & 8,00 & 7,89 & 8,00 & 8,04 & 8,00 \\
\hline $\begin{array}{l}\text { F) Satisfacción general y aprendizajes } \\
\text { logrados }\end{array}$ & 8,17 & 9,00 & 7,95 & 9,00 & 8,05 & 9,00 \\
\hline
\end{tabular}

Fuente: Elaboración propia.

Las puntuaciones medias para cada dimensión arrojan resultados notablemente altos -entre 7.08 y 8.79 en una escala Likert de 0 a 10 puntos- lo que lleva a interpretar que la satisfacción del profesorado con la formación en ambas modalidades es notoria. Se verifica que la tendencia apuntada por las medias globales se repite; sin embargo, se aprecian valores ligeramente mayores en la modalidad de autoformación como ya apuntó el análisis pormenorizado de los ítems. Para determinar si estas diferencias son estadísticamente significativas, se ha realizado un análisis inferencial, partiendo de la comparación de las diferencias observables.

En primer lugar, se realizó la prueba de normalidad, analizando el estadístico Kolmogorov-SmirnovLilliefors se comprobó que el estadístico de contraste era menor que 0,05 , en concreto era $p=0$ en todas las dimensiones, por lo que al no detectarse normalidad en la prueba se acordó aplicar el estadístico no paramétrico de Mann-Whitney:

TABLA 5. RESULTADOS DEL TEST NO PARAMÉTRICO DE MANN-WHITNEY

\begin{tabular}{lcccccc}
\hline & A. & B. & C. & D. & E. & F. \\
\hline U de Mann-Whitney & $282.627,000$ & $288.477,000$ & $241.942,500$ & $290.551,500$ & $284.832,000$ & $295.416,000$ \\
\hline Z & -3.880 & -3.304 & -8.320 & -3.082 & -3.726 & -2.534 \\
\hline Sig. asintót. (bilateral) & .000 & .001 & .000 & .002 & .000 & .011 \\
\hline
\end{tabular}

Fuente: Elaboración propia.

Dado que el p-valor asociado al estadístico del contraste de medianas es inferior a 0.05 -para un nivel de confianza del $95 \%$ - en todos los casos, se puede establecer que todas las comparaciones muestran di- 
ferencias estadísticamente significativas, o lo que es lo mismo que se rechaza la hipótesis nula de igualdad de medianas, en todos y cada uno de los Test de la $U$ de Mann-Whitney.

Por otro lado, partiendo de que descriptivamente se comprueba que existe una diferencia entre las medias de las diferentes modalidades y teniendo en cuenta que la muestra se considera lo suficientemente elevada ( $N=1596$ ), a pesar de que no se cumple el supuesto de Normalidad, se ha decidido aplicar la prueba paramétrica de la $t$ de Student que posibilita comparar las medias.

tABla 6. Resultados de LA PRUEba PARAMÉtrica t de STUdent

\begin{tabular}{|c|c|c|c|c|c|c|c|}
\hline & & A. & B. & C. & D. & E. & F. \\
\hline \multirow{2}{*}{$\begin{array}{l}\text { Prueba de Levene para } \\
\text { la igualdad de varianzas }\end{array}$} & $\mathbf{F}$ & 8,606 & 1,022 & 27,427 & 8,409 & 0,015 & 1,544 \\
\hline & Sig. & 0,003 & 0,312 & 0 & 0,004 & 0,902 & 0,214 \\
\hline \multirow{2}{*}{$\begin{array}{l}\text { Prueba t para } \\
\text { la igualdad de medias }\end{array}$} & $T$ & 3,884 & 2,86 & 8,056 & 2,936 & 3,049 & 2,205 \\
\hline & $\begin{array}{c}\text { Sig. } \\
\text { (bilateral) }\end{array}$ & 0 & 0,004 & 0 & 0,003 & 0,002 & 0,028 \\
\hline
\end{tabular}

Fuente: Elaboración propia.

Tanto la prueba de Mann-Whitney como la $t$ de Student (véanse tablas 5 y 6 ) apuntan a que las 6 dimensiones del cuestionario sí muestran diferencias estadísticamente significativas entre las modalidades de formación estudiadas, ya que todas ofrecen un $p$-valor menor que 0.05 , es decir, que las respuestas a los diferentes ítems sí dependen de la modalidad formativa de que se trate.

A continuación, se ha procedido a analizar las dimensiones del cuestionario argumentando las diferencias de las medias observables que, en todo caso se aprecian más altas en autoformación que en presencial, como se viene reiterando.

En primer lugar, con respecto a la evaluación de la organización, estos resultados pueden explicarse considerando que en FC y GT la formación tiene lugar en el centro educativo lo que evita desplazamientos a lugares ajenos al participante y propicia un acceso fácil a los recursos que ofrece el entorno de trabajo. Asimismo, el calendario de reuniones y ponencias es consensuado por el grupo y cada individuo decide los momentos de su proceso de investigación-acción, concediendo una mayor adaptabilidad y conciliación familiar. En contraposición, la presencialidad tiene una organización más rígida porque tanto la agenda como el lugar es impuesto de antemano desde los CEP.

En la dimensión contenidos de la formación, el motivo de que perciban con mayor interés para la docencia los contenidos trabajados radica en que en autoformación se diseña un proyecto en común, partiendo de una misma realidad, con una finalidad y unos contenidos contextualizados que dan respuesta a necesidades formativas concretas. En cuanto a la dificultad de los contenidos, en general, la mayor parte los interpretan como de dificultad media, lo que supone su adecuación a la heterogeneidad de los niveles de competencia profesional encontrados.

En lo concerniente al desarrollo de la formación, dimensión en la que se miden aspectos como el fomento del trabajo colaborativo, el nivel de interacción entre individuos y la idoneidad de la metodología utilizada, la justificación se encuentra en que el desarrollo de la formación tiene lugar en el centro a través del trabajo conjunto de sus integrantes, generando un sistema compartido compuesto por individuos en continua interacción que acomodan en grupo su desarrollo profesional, lo que en efecto provoca que la percepción sobre la idoneidad de la metodología utilizada sea mayor.

Las ponencias también registran valores ligeramente más altos en autoformación, a pesar de que en este caso representan solo el $15 \%$ de las horas de formación certificables. La explicación está en que esta modalidad tiene como punto de partida las necesidades formativas de un grupo con una misma finalidad, esto hace que los ponentes sean más operativos adecuando sus exposiciones y sus propuestas a dichas necesidades.

En lo referente a las asesorías, es reseñable que el profesorado reconozca su labor en el diseño de actividades y en la resolución de incidencias; de nuevo confirmamos una tendencia mayor en autoformación. La explicación la hallamos en que la asesoría programa el proyecto de autoformación junto al 
claustro adecuándolo al centro y hace un seguimiento de este durante todo el curso académico lo que provoca que sean percibidos de manera ostensible. Mientras que en CP el diseño parte de un análisis de necesidades general sin tener en cuenta a los asistentes y el seguimiento tiene menor percepción por parte de estos.

Por último, en relación con la satisfacción general y los aprendizajes logrados, la autoformación vuelve a registrar valores algo superiores; la adecuación de la formación a las expectativas iniciales es debida que son los propios formados quienes la programan. Esta conlleva un proceso investigación-acción que sitúa al docente como un profesional reflexivo que convierte su puesto de trabajo en un laboratorio en el que investiga y reflexiona sobre la práctica con el propósito de aprender. El nivel de aprendizaje alcanzado está relacionado con la justificación anterior: en la autoformación los aprendizajes son más significativos en la medida en que estos tienen lugar en un contexto propio partiendo de los conocimientos y la experiencia de un grupo con una finalidad común. Indudablemente, todo lo anterior repercute en un notable nivel de satisfacción lo que se traduce en recomendar a otros participar en actividades similares.

\subsection{Preguntas abiertas del cuestionario}

Las preguntas abiertas proporcionan un gran volumen de información valiosa y relevante para entender y matizar los datos cuantitativos. No obstante, su tratamiento supone una tarea ardua por las dificultades de su análisis. Para poder interpretar y comparar las respuestas se procedió a su lectura con la finalidad de poder establecer categorías acordes con los criterios temáticos del presente estudio y con las propias contestaciones recopiladas. Definidas dichas categorías, se contabilizó la frecuencia con la que los participantes las reseñaron y se determinó el porcentaje (\%) de individuos de cada muestra que las señaló. Finalmente, se analizaron los resultados como se recoge en los párrafos siguientes.

A la pregunta «¿Cuáles son los aspectos de la formación que mejor has valorado?» se obtenían las respuestas recogidas en la figura 1 :

FIGURA 1. ASPECTOS MEJOR VALORADOS DE LA FORMACIÓN

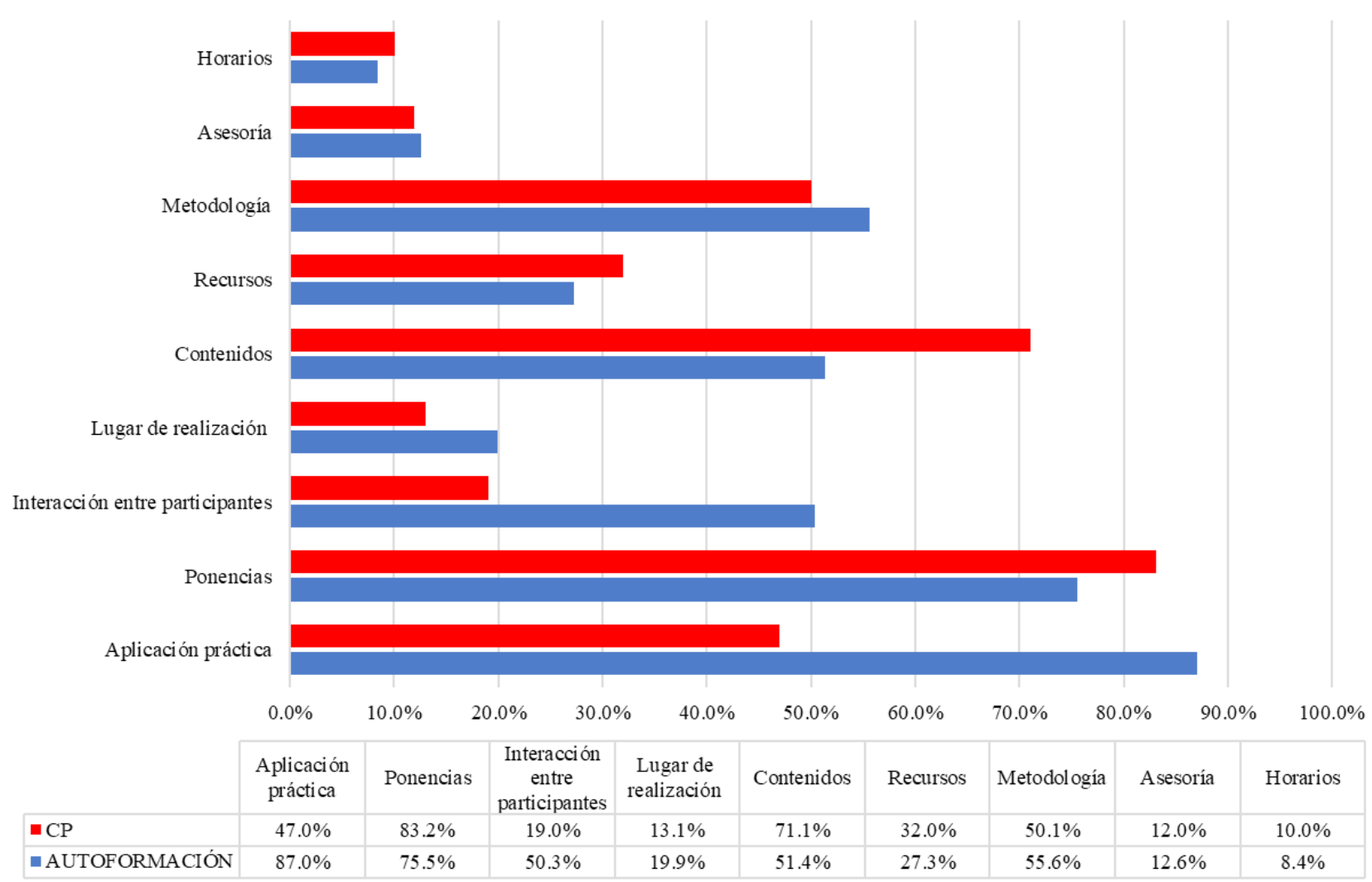

Fuente: Elaboración propia. 
Con respecto a la aplicación práctica, en ambas modalidades los participantes consideran que entre los aspectos mejor considerados está la utilidad de la formación, es decir, que el aprendizaje logrado pueda ser aplicable al puesto de trabajo. En autoformación se verifica que la mayoría $(87,0 \%)$ lo señala como el aspecto más palpable y mejor valorado; es una modalidad que tiene lugar en el centro educativo a lo largo de todo el curso académico y donde los docentes se erigen como investigadores de su propia acción, estos experimentan y aprenden aplicando en el aula los nuevos conocimientos que van adquiriendo, lo que hace que estén más satisfechos con este aspecto de la formación. En CP la aplicación práctica es percibida con menor importancia, no obstante, casi la mitad $(47,0 \%)$ valoró positivamente haber aplicado lo aprendido; la interpretación de esta diferencia está en que, por un lado, en CP la aplicación en el puesto de trabajo no forma parte del aprendizaje y, por otro, en que los formados desechan la oportunidad de llevar al aula la formación cuando evidencian que las prácticas ofrecidas por los ponentes no son replicables en sus contextos laborales.

Destacan también las ponencias, en CP constituye la categoría mejor valorada $(83,2 \%)$ debido a que el ponente es el eje rector de esta formación, muestra sus experiencias didácticas, establece pautas y plantea tareas, y posibilita la resolución inmediata de dudas. En autoformación es el segundo factor mejor valorado $(75,5 \%)$ tras la aplicación práctica, esta modalidad cuenta con un apoyo formativo externo que puede llegar a constituir el $15 \%$ de las horas certificables de la formación y cuyo objetivo es facilitar la adquisición de aquellos conocimiento que los participantes de FC o GT no pueden adquirir a través de los procesos de investigación, reflexión y puesta en práctica que desarrollan; de acuerdo con las respuestas recogidas, las ponencias externas generan inercias muy positivas para impulsar la formación y asegurar su funcionamiento.

Las respuestas relativas a la metodología aparecen siempre vinculadas a los contenidos y a los ponentes. En autoformación algo más de la mitad $(55,6 \%)$ de los participantes valoran positivamente este aspecto de la formación y destacan, como características positivas, que sea activa, participativa, colaborativa, dinámica y centrada en la práctica. En CP la metodología es apreciada por la mitad (50,1\%), los ponentes adecuan la metodología en función de los contenidos con la finalidad de facilitar la interacción con los participantes, facilitar la teoría, resolver y debatir problemas prácticos y compartir experiencias.

Los contenidos deben responder a las necesidades formativas de los participantes, establecen la base para generar las competencias profesionales del docente y aparecen en la combinación de teoría y práctica facilitando el proceso de aprendizaje. En CP un amplio porcentaje $(71,1 \%)$ apreció positivamente este factor. En autoformación, aunque son apreciados por la mitad $(51,4 \%)$ de los participantes, el que lo hagan en menor medida que en CP respondería a que otorgan mayor importancia a la aplicación práctica de dichos contenidos que a estos per se.

Conforme a las respuestas analizadas, en autoformación la interacción entre participantes es valorada positivamente por la mitad $(50,3 \%)$ de los sujetos y tiene lugar a través del trabajo colaborativo; la construcción de nuevos conocimientos surge del trabajo en equipo por lo que cualquier tarea que realicen se verá enriquecida y sustentada por la capacidad, la experiencia y la práctica de sus integrantes. No obstante, algunos de ellos admiten que el trabajo colaborativo a pesar de ser netamente enriquecedor también conlleva ciertas trabas como la sensación de pérdida de tiempo y de menor rendimiento al tener que definir estrategias y alcanzar acuerdos en equipo, la imposición de criterios por parte de algunos sujetos y la aparición de subgrupos. En CP esta interacción $(19,0 \%)$ se percibe de modo diferente, más como una forma de socializar y compartir experiencias entre participantes y en torno a la temática del curso, ya que en un mismo espacio y tiempo pone en contacto a docentes con inquietudes similares, pero con un origen diferente.

En cuanto a los recursos, tienen desigual consideración entre una y otra modalidad, aunque el porcentaje de formados que los destacan sea muy similar. En CP (32,0\%) los recursos son ofrecidos principalmente por los ponentes o la administración para el desarrollo del curso; en cambio, en autoformación $(27,3 \%)$ si bien también se aprecian los recursos facilitados por los ponentes, los más valorados son los que se van generando como parte de las tareas del propio proceso de formación.

Las respuestas dadas con relación al lugar de realización también difieren en ambas modalidades. En autoformación (19,9\%) se aprecia que la formación se produzca en el centro educativo, contextualizándose y evitando desplazamientos a lugares ajenos al sujeto. En CP $(13,1 \%)$, se valora que la formación tenga lugar en espacios no formales de aprendizaje como extensión y alternativa a las instalaciones del CEP: museos, universidades, espacios naturales y al aire libre.

La labor de la asesoría es observada y apreciada por su presencia en todo el proceso en autoformación $(12,6 \%)$. En la misma medida en CP $(12,0 \%)$ aunque los asistentes no perciben su presencia, pero sí reconocen su papel en el diseño y organización de la actividad. 
Por último, los horarios, es el factor apreciado en menor grado y las razones de su consideración divergen de una a otra modalidad. En CP $(10,0 \%)$ se valora que el calendario se fije de forma previa a la realización de la actividad, de manera que los sujetos pueden planificarse de antemano. En autoformación $(8,4 \%)$ se valora la adaptabilidad a los horarios docentes, para el trabajo individual cada uno selecciona los momentos que dedicará a la formación y en el caso del trabajo en equipo se hace coincidir con los horarios de permanencia en el centro.

A la pregunta «¿Cuáles son los aspectos que podrían mejorar?» se obtienen los resultados incluidos en la figura 2:

FIGURA 2. ASPECTOS DE LA FORMACIÓN QUE PODRÍAN MEJORAR

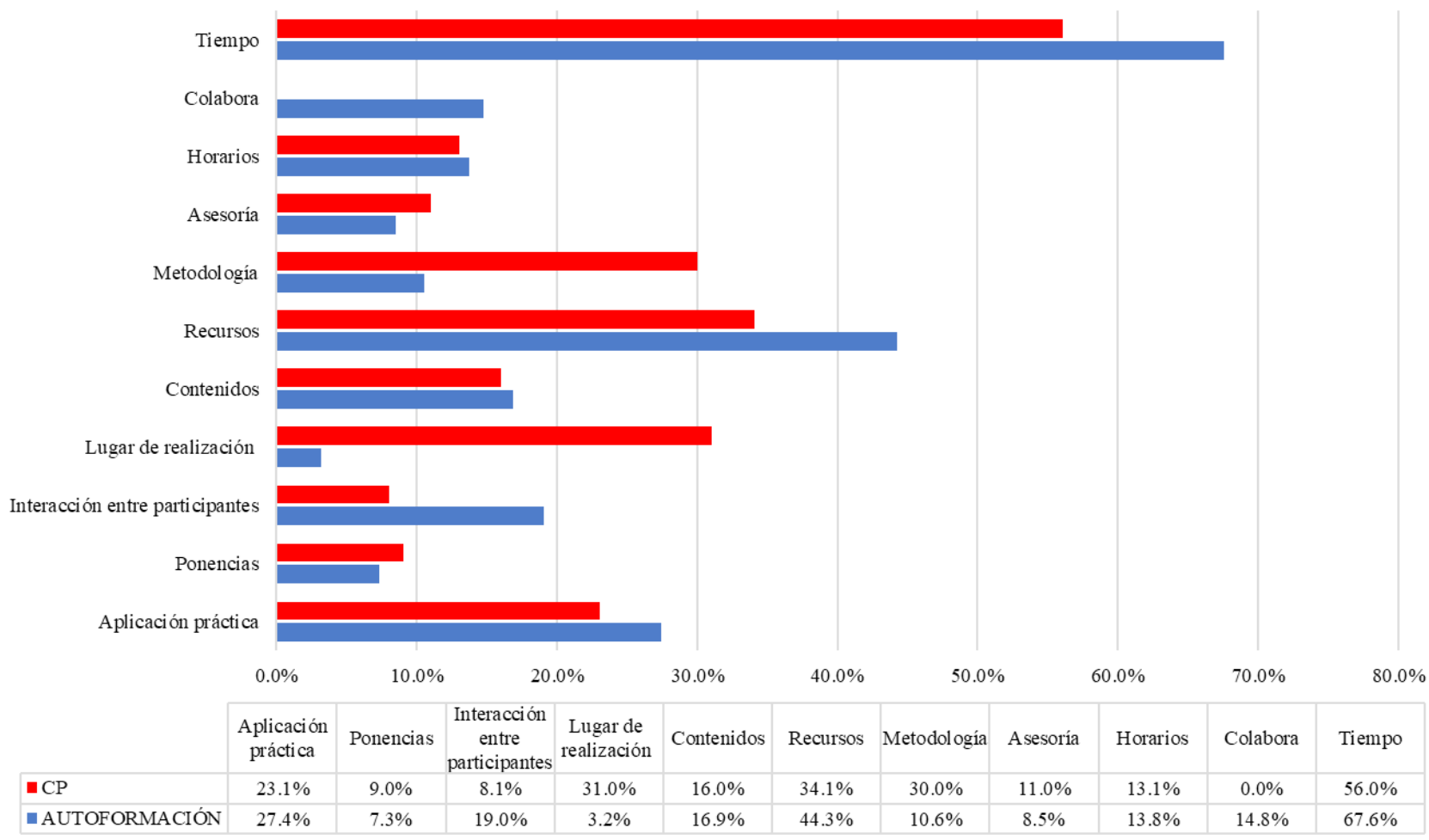

Fuente: Elaboración propia.

El aspecto a mejorar que con más frecuencia señalaron los participantes, es la falta de tiempo, pero los argumentos difieren de una modalidad a otra. En autoformación los encuestados declaran que necesitan más tiempo $(67,6 \%)$ ya que el proceso de aprendizaje supone un esfuerzo añadido a las muchas obligaciones en las que se ven envueltos a lo largo del curso, lo que les genera una sensación de desasosiego; posiblemente se deba a que se plantean muchos objetivos para el periodo de formación previsto y disponen de lapsos de tiempo muy limitados para alcanzarlos, es decir, para dedicar a la formación, al trabajo conjunto, a la reflexión compartida y a la aplicación; además, demandan la ampliación de las horas destinadas al apoyo formativo externo. Las respuestas dadas en CP $(56,0 \%)$ hacen referencia a la necesidad de ampliar las sesiones de formación debido a que les falta tiempo para asimilar los contenidos; el origen del problema se encuentra en que este tipo de actividades trata de ofrecer a los asistentes la máxima información sobre una temática dada en un determinado número de sesiones, pero no todos la asimilan al mismo ritmo ya que los grupos están integrados por individuos con distinto origen y nivel de competencia, y en ocasiones, o bien no da tiempo a ofrecer todos los contenidos o a algunos integrantes no les da tiempo a asimilarlos.

Por lo que atañe a los recursos tanto en autoformación (44,3\%) como en CP $(34,1 \%)$ las respuestas de los participantes revelan las consecuencias de la falta de inversión en educación de los últimos años. La disponibilidad de recursos para formación en los CEP (materiales, tecnológicos, técnicos y humanos) es mayor que en los centros educativos ordinarios, es por ello por lo que la necesidad de recursos es más patente en autoformación. 
La aplicación práctica registra valores muy similares tanto en CP $(23,1 \%)$ como en autoformación $(27,4 \%)$, en esta última modalidad es donde mejor se valora la aplicación práctica de la formación, como se observa en la figura 1, por lo que probablemente esto explica que su valor sea ligeramente más alto que en CP, debido a que los participantes son más conscientes de la necesidad de poner en práctica los conocimiento adquiridos; esta preocupación respaldaría que un número mayor de participantes lo consideren como un a aspecto a mejorar.

Un razonamiento similar al anterior justifica que el valor registrado en la necesidad de mejorar la interacción entre participantes sea mayor en autoformación (19,0\%), dado que los docentes son más conscientes de dicha necesidad en esta modalidad que en la de CP $(8,1 \%)$, sobre todo porque la colaboración entre compañeros es un aspecto fundamental para garantizar el éxito de las FC y los GT, véase figura 1.

En cuanto a las respuestas recopiladas en torno a los contenidos son muy similares tanto en CP $(16,0 \%)$ como en autoformación $(16,9 \%)$ haciendo referencia a que los contenidos sean más concretos y se ajusten a las necesidades educativas del profesorado.

Llama la atención que se ha reducido el porcentaje de participantes $(14,8 \%)$ contrarios a Colabora (entorno virtual de trabajo bajo el modelo de las webs 2.0 que ofrece un espacio de aprendizaje colaborativo y cuyo uso es obligado únicamente en las modalidades de autoformación). El uso de esta plataforma suscitó mucho rechazo en sus comienzos, y con las respuestas recogidas se confirma que aún hoy en día todavía un pequeño porcentaje de participantes proponen la eliminación del uso de esta plataforma como elemento de mejora.

A propósito de los horarios, en las dos opciones formativas son considerados en similar proporción, pero los argumentos de mejora dados son diferentes. En autoformación $(13,8 \%)$ se reclama fijar de antemano un horario evitando sus modificaciones; los cambios de fecha en este tipo de modalidad suelen ser bastante frecuentes debido a que la proximidad entre los participantes permite atender a las diferentes casuísticas que acontecen en el centro, posponiendo o adelantando reuniones que, si bien se realizan en base al interés general, ello no evita que algunos individuos resulten agraviados. En otro orden de ideas, en CP $(13,1 \%)$ se reclama que los horarios de formación se integren dentro de las horas lectivas, adaptando la jornada laboral para permitir la asistencia a cursos de formación; también, se reclama la compatibilidad entre la asistencia a los citados cursos y el trabajo, dado que la mayoría de las formaciones tienen lugar en horario de tarde excluyendo de la oferta al profesorado que trabaja en esa franja (conservatorios, escuelas de idiomas, etc.).

Respecto a la metodología, al analizar las respuestas observamos que los participantes de CP $(30,0 \%)$ son los menos satisfechos con este aspecto, entre sus inconvenientes señalan: algunos ponentes transmiten sus conocimientos de forma unidireccional no empleando metodologías activas y participativas que involucren a los formados; el abuso de la transmisión por vía oral provoca que algunos contenidos se pierdan; se frena el pensamiento crítico en los formados; $y$ en los grupos muy numerosos se pierde la atención causando aburrimiento y distracción. En autoformación (10,6\%) las mejoras reclamadas están en la línea de mejorar el trabajo cooperativo y la puesta en práctica de ejemplos reales.

En cuanto a las asesorías desde la CP $(11,0 \%)$, donde son menos visibles, el profesorado solicita su presencia y que estas realicen un mayor seguimiento de sus progresos. Igualmente, en autoformación $(8,5 \%)$ se demanda que exista un mayor seguimiento y feed-back por parte de las asesorías.

Las mejoras demandadas en relación con las ponencias, tanto en autoformación $(7,3 \%)$ como en CP $(9,0 \%)$, hacen referencia a que los ponentes se centren en la práctica y desarrollen dinámicas activas que impliquen a todos los participantes.

Por último, en referencia al lugar de realización, comprobamos la gran diferencia entre autoformación $(3,2 \%)$ debido a que solo un reducido número de docentes no considera positivo que se realice en su lugar de trabajo; y CP $(31,0 \%)$ donde los participantes desaprueban las instalaciones del CEP de Málaga y destacan la dificultad para desplazarse hasta esta institución, sobre todo en el caso de los municipios más alejados de la capital.

\section{CONCLUSIONES}

Las conclusiones de esta investigación realizada en el CEP de Málaga permiten considerar aspectos que pueden facilitar la toma de decisiones, a nivel político y técnico, en cuanto a las modalidades formativas que se deben promover desde este tipo de instituciones. Los resultados obtenidos ameritan su difusión en el resto de CEP de Andalucía e incluso en el ámbito nacional. Si bien la ordenación de la educación permanente en el marco nacional posee sus propias especificidades, en todo el territorio se comparte la necesidad de optimizar los limitados recursos económicos destinados a la formación continua. A pesar de que la autoformación po- 
dría suponer menores costes que la formación presencial, es necesario asegurar que este modelo formativo reporte efectos positivos al profesorado y por consiguiente a las organizaciones que lo implementan.

Tras analizar los datos obtenidos con las respuestas cerradas, se observa que muestran diferencias estadísticamente significativas dependiendo de la opción seleccionada y se verifica que todas las valoraciones son ligeramente más altas en autoformación. La evaluación de la organización de la actividad en esta modalidad está caracterizada por que es el individuo junto con el grupo el que decide los momentos que dedicará a su proceso de aprendizaje, el cual tiene lugar en un contexto próximo como es el propio centro educativo que le brinda recursos formativos inmediatos y accesibles. Los objetivos y los contenidos son recogidos en un proyecto que parte del análisis conjunto de la realidad para dar respuesta a unas necesidades formativas concretas. Por tanto, esto permite que la actividad responda a las expectativas iniciales de los participantes, quienes la consideran esencial para mejorar su práctica profesional. Este tipo de formación propicia la interacción entre los sujetos y saca al docente del ostracismo de su aula para compartir inquietudes con sus iguales, potenciando el trabajo colaborativo y desplegando una metodología activa y cooperativa que los involucra a todos. En la investigación de Pérez (2018) sobre autoformación, se demuestra que las metodologías que promueven el aprendizaje autónomo, horizontal y colaborativo resultan más atractivas y más efectivas para el cambio de las prácticas educativas. Por otro lado, el apoyo formativo dado a la autoformación genera una inercia muy significativa en el proceso de investigación-acción e impulsa al grupo a superar los escollos que frenan su progreso, dando feed-back sobre la ejecución y propiciando el aprendizaje activo y la resolución de problemas. La asesoría es percibida con mayor cercanía debido a un contacto constante que se inicia con la redacción del proyecto, prosigue con un seguimiento de su desarrollo y finaliza con el reconocimiento del proceso seguido por cada docente. Como refiere Imbernón (1996) esta modalidad de formación permite que los profesores alcancen sus objetivos y satisfagan sus necesidades de aprendizaje, ya que el docente aprende utilizando como estrategias formativas la confrontación reflexiva de su práctica en un entorno compartido con sus iguales. Todo ello repercute en un mayor grado de satisfacción y en consecuencia los docentes recomendarían esta modalidad a otros.

A la luz de los resultados obtenidos con las preguntas abiertas, llegamos a la conclusión de que la administración educativa, en materia de formación del profesorado, debe: facilitar recursos y tiempo a sus docentes para permitir su desarrollo profesional a través de la formación; diseñar actividades con transferencia al puesto de trabajo; contratar ponentes que desarrollen metodologías dinámicas y participativas; seleccionar contenidos que respondan a las necesidades formativas de los docentes; favorecer el trabajo colaborativo entre los sujetos y el intercambio de experiencias; acercar la formación a los centros educativos para que cobren protagonismo como espacios para el aprendizaje docente; y asegurar el seguimiento y tutorización de los progresos del profesorado durante la formación.

Como ya hemos apuntado anteriormente, esta investigación respalda la adecuada dirección de las políticas adoptadas en educación permanente del profesorado en Andalucía, la autoformación constituye la opción con mejor valoración por parte de quienes deciden realizar algún tipo de formación, la necesidad de los profesores de desarrollarse profesionalmente surge no sólo a partir de inquietudes personales sino también de la necesidad de resolver grupalmente los problemas de su contexto escolar. No obstante, la formación presencial también obtiene notables valoraciones lo que nos debe llevar no solo a defenderla sino a mejorarla. En este sentido establece Vezub (2015) que los docentes cuando seleccionan una modalidad u otra manifiestan su particular modo de entender la formación, de cubrir sus intereses y las necesidades individuales y grupales de su contexto inmediato.

Con el propósito de seguir desarrollando esta línea de investigación se está trabajando en un análisis comparativo entre ambas modalidades, en relación con la transferencia, para comprobar la eficacia de cada una, identificando en qué medida los aprendizajes logrados con la formación se evidencian en la práctica docente una vez que finaliza y determinando qué factores favorecen u obstaculizan el proceso. Otra línea de investigación futura reside en determinar qué aspectos del diseño y desarrollo de una formación aseguran una mayor eficacia en la transmisión de aprendizajes al puesto de trabajo.

\section{REFERENCIAS BIBLIOGRÁFICAS}

ANEAS, C., SÁNCHEZ, J. \& SÁNCHEZ, E. (2018): "Diseño y validación de dos cuestionarios dirigidos a evaluar la eficacia de la formación del profesorado", en Docencia e Investigación, núm. 28, págs. 50-76. Recuperado de https://revista.uclm.es/index.php/rdi/article/view/1715. 
BANDEIRA, W. (2001): "Factores caracterizadores de centros educativos eficaces”, en Bordón, vol. 53, núm. 2, págs. 175-184

BARQUÍN, J. (2014): Informe sobre el profesorado de Andalucía y la formación permanente. Informe Inédito. EVACENPRO (Estudio sobre la evaluación de los centros de Andalucía). Junta de Andalucía.

COSTA, A. (2015): "Los movimientos de renovación pedagógica, las reformas y la formación del profesorado en España", en Reformas educativas y formación de profesores, págs. 171-194. Madrid: Biblioteca Nueva.

Decreto 93/2013, de 27 de agosto, por el que se regula la formación inicial y permanente del profesorado en la Comunidad Autónoma de Andalucía, así como el Sistema Andaluz de Formación Permanente del Profesorado. Boletín Oficial de la Junta de Andalucía, núm. 170 de 30 de agosto de 2013, págs. 6-50. https://www. juntadeandalucia.es/boja/2013/170/BOJA13-170-00142.pdf.

DÍAZ, L. A. (2016): "Políticas de la Unión Europea en materia de formación continua del profesorado", en CARRILLO, I. (ed.): Democracia y Educación en la formación docente, vol. 1, págs. 305-311. Barcelona: Universitat de VicUniversitat Central de Catalunya. Recuperado de https://dialnet.unirioja.es/servlet/articulo?codigo=5789084.

ELLIOT, J. (2010): "El «estudio de la enseñanza y del aprendizaje»: una forma globalizadora de investigación del profesorado", en Revista interuniversitaria de formación del profesorado, núm. 68, págs. 223-242. Recuperado de https://dialnet.unirioja.es/servlet/articulo?codigo=3276062.

Estadísticas - Recursos Humanos del Sistema Educativo en Andalucía - Consejería de Educación. (2018, septiembre). Recuperado 27 de octubre de 2018, de http://www.juntadeandalucia.es/educacion/webportal/web/estadisticas/ recursos-humanos-del-sistema-educativo-en-andalucia/-/libre/detalle/14Li/datos-avance-2017-2018-menurecursos-humanos.

GARcíA, E., COlÉN, M. T., MAuRI, T., RABAdÁN, J. M., MELlAdO, V., PÉREZ, J. I. F., HERNÁNDEZ, I. C. (2001): La formación del profesorado: Proyectos de formación en centros educativos. Barcelona: Grao.

HERNÁNDEZ, R., FERNÁNDEZ, C. \& BAPTISTA, P. (2014): Metodología de la Investigación (6. ${ }^{a}$ edición). México: McGraw Hill.

IMBERNÓN, F. (1996): "La formación en los centros educativos: ¿tendencia o moda?", en Aula de innovación educativa, núm. 46, págs. 43-46.

IMBERNÓN, F. (2002): "Reflexiones globales sobre la formación y el desarrollo profesional del profesorado en el Estado español y Latinoamérica”, en Educar, núm. 30, págs. 15-25.

IMBERNÓN, F. (2006): "Actualidad y nuevos retos de la formación permanente”, en Investigación educativa, vol. 8, núm. 2, págs. 1-11. Recuperado de http://redie.uabc.mx/vol8no2/contenido-imbernon.html.

IMBERNÓN, F. (2012): “Un nuevo desarrollo profesional del profesorado para una nueva educación”, en Ciencias Humanas, vol. 12, núm. 19, págs. 75-86. Recuperado de http://www.revistas.fw.uri.br/index.php/revistadech/ article/view/343.

IMBERNÓN, F. (2017): Ser docente en una sociedad compleja: La difícil tarea de enseñar. Barcelona: Grao.

Instrucción 17/2017, de 1 de septiembre, de la Dirección General del Profesorado y Gestión de Recursos Humanos para el desarrollo de la formación en centro para el curso 2017/2018. http://www.adideandalucia.es/normas/ instruc/Instruccion17-2017FormacionCentros17-18.pdf.

Instrucción 18/2017, de 1 de septiembre, de la Dirección General del Profesorado y Gestión de Recursos Humanos para el desarrollo de grupos de trabajo para el curso 2017/2018. http://www.adideandalucia.es/normas/instruc/ Instruccion18-2017GruposTrabajo17-18.pdf.

JARAUTA, B., COLÉN, M. T., BARREDO, B. \& BOZU, Z. (2014): "La formación permanente del profesorado en Cataluña: análisis de los referentes legales”, en Bordón, vol. 66, núm. 4, págs. 87-101. DOI: https://doi. org/10.13042/bordon.2014.66407.

MARCELO, C. (1999): Formación del profesorado para el cambio educativo. Barcelona: Promociones y Publicaciones Universitarias, PPU.

MARTÍN, E. (2005): La formación en centros: un modelo de formación permanente para equipos docentes. Valencia: Nau Llibres.

MURILLO, F. J. \& KRICHESKY, G. J. (2018): "La colaboración docente como factor de aprendizaje y promotor de mejora. Un estudio de caso”, en Educación XXI, vol. 21, núm. 1, págs. 135-156. DOI: https://doi.org/10.5944/ educxx1.20181.

Orden de 31 de julio de 2014, por la que se aprueba el III Plan Andaluz de Formación Permanente del Profesorado. Boletín Oficial de la Junta de Andalucía, núm. 170 de 2 de septiembre de 2014, págs. 9-34. https://www. juntadeandalucia.es/boja/2014/170/BOJA14-170-00117.pdf.

PÉREZ, Á. (2017): Pedagogías para tiempos de perplejidad. De la información a la sabiduría. Rosario: Homo Sapiens.

PÉREZ, L. (2018): "El grupo de trabajo como estrategia de formación permanente del profesorado", en Aula de Encuentro, vol. 20, núm. 1, págs. 4-25. DOI: https://dx.doi.org/10.17561/ae.v20i1.1.

PÉREZ, R., LÓPEZ, F., PERALTA, M. D. \& MUNICIO, P. (2000): Hacia una educación de calidad: Gestión, instrumentos y evaluación. Madrid: Narcea Ediciones.

Resolución de 12 de septiembre de 2017, de la Dirección General de Profesorado y Gestión de Recursos Humanos, por la que se determina el desarrollo de las líneas estratégicas de formación del profesorado establecidas en el III Plan Andaluz de Formación Permanente del Profesorado y la elaboración de los proyectos de formación para el 
curso 2017/18. Boletín Oficial de la Junta de Andalucía, núm. 183 de 22 de septiembre de 2017, págs. $120-123$. https://www.juntadeandalucia.es/boja/2017/183/BOJA17-183-00004-16056-01_00121177.pdf.

SANTOS, M. Á. (2010): "La formación del profesorado en las instituciones que aprenden", en Interuniversitaria de formación del profesorado, núm. 68, págs. 175-200. Recuperado de https://dialnet.unirioja.es/servlet/ articulo?codigo $=3276058$.

VALLE, J. M. (2017): “La formación inicial del profesorado según la Unión Europea”, en Nueva Revista, núm. 141, págs. 32-50. Recuperado de https://reunir.unir.net/handle/123456789/5235.

VEZUB, L. F. (2013): "Hacia una pedagogía del desarrollo profesional docente. Modelos de formación continua y necesidades formativas de los profesores", en Páginas de Educación, vol. 6, núm. 1, págs. 97-124. DOI: https:// doi.org/10.22235/pe.v6i1.535. 